International Journal of Biology, Pharmacy and Allied Seiences (IJBPAS) 'A Bridge Betuen Caboratory and QRando'

WwW.iibpas.com

\title{
SYNTHESIS AND EVALUATION OF ISATIN DERIVATIVES AS
}

\section{ANTIMICROBIALS}

\author{
YADAV $M^{1,2^{*}}$, KUMAR $S^{2}$ AND HUSAIN $A^{3}$ \\ ${ }^{1}$ AVIPS, Shobhit University, Gangoh, Saharanpur-247341 (U.P.) India \\ ${ }^{2}$ School of Pharmaceutical Sciences, IFTM University, Moradabad-244102 (U.P) India \\ ${ }^{3}$ Faculty of Pharmacy, Jamia Hamdard University, New Delhi-110019, India
}

*Corresponding Author: E Mail: mayankmp@gmail.com

Received 18 ${ }^{\text {th }}$ Oct. 2019; Revised 13 ${ }^{\text {th }}$ Nov. 2019; Accepted 22 ${ }^{\text {nd }}$ Dec. 2019; Available online $1^{\text {st }}$ April 2020

\section{https://doi.org/10.31032/IJBPAS/2020/9.4.5006}

\section{ABSTRACT}

With the findings of bioactivity of isatin derivatives, present investigation aim to synthesize new isatin derivatives (PD1-15) and evaluation for their antimicrobial activity. The isatin derivatives were obtained by reaction of istain (1) with p-phenylenediamine (2) in ethanol to afford 3-(4-aminophenylimino)-indolin-2-one (3) on chloroacetylation to afford the N-(4-(2oxoindolin-3-ylideneamino)-phenyl)-2-chloroacetamide (4) which further reacted with different phenols and primary amines in presence of anhydrous potassium carbonate in acetonitrile. The test compounds characterized by spectroscopic methods. All the test compounds were screened for their antimicrobial activity against Staphylococcus aureus, Bacillus subtilis (Gram-positive bacteria), Escherichia coli, Klebsiella pneumoniae (Gramnegative bacteria) and Candida albicans, Aspergillus niger, Penicillium chrysogenum, Rhizopus oryzae (fungal strains) by cup-plate method by measuring zone of inhibition. The MIC value of potent test compounds was also determined. Molecular properties of test compounds were determined by online software. The results showed isatin derivatives may be optimized as lead compound as antimicrobials.

Keywords: Isatin; Antimicrobials; Molecular properties, Phenols, Amines

\section{INTRODUCTION}

There are several heterocyclic compounds but five and six membered ring compounds have gained a special place among different classes of organic compounds for their 
versatile and diversified biological activities [1]. Among all the variety of heterocycles that have been identified for producing pharmaceutically valuable molecules, indoles and oxadiazines have shown a remarkable role in pharmaceutical chemistry $[2,3]$. Isatin is an indole derivative containing keto groups at 2- and 3- positions. Previous studies on the action of oxidizing agents on indigo lead to the discovery of an oxidation product to which the name isatin was given [4].

Isatin is an endogenous compound reported in many organisms, performs a wide range of biological activities [5]. The isatin ring system is a versatile structural design found in various biologically active compounds. This is mainly due to the easy synthesis and the importance of pharmacological activity. Therefore, the synthesis and selective fictionalization of isatin has been the main emphasis of active research area over the years [6]. Isatin derivatives are well reported for their antibacterial [7] and antifungal [8] activity. The search for novel antimicrobial agents is a challenging task for researchers because of unexpected increasing the resistance of microbial pathogens and it is in demand to search the drugs with improved potency and broader activity spectrum $[9,10]$. By keeping the above considerations, the present research work is aimed to synthesize new isatin derivatives and show promising antimicrobial activity.

\section{MATERIALS AND METHODS}

All solvents used were of laboratory grade and were obtained from $\mathrm{CDH}$ and SD fine chemicals. Melting points of the synthesized compounds were determined by open capillary method and are uncorrected. The IR spectra of synthesized compounds were recorded in potassium bromide discs on FTIR Bruker (Bruker) Spectrophotometer. The ${ }^{1} \mathrm{H}$ NMR spectra were recorded on a AVANCE III (Bruker) NMR Spectrophotometer at $512 \mathrm{MHz}$ using $\mathrm{CDCl}_{3}$ as a solvent and TMS as internal standard. All chemical shift values are reported in $\operatorname{ppm}(\delta)$. The reactions progress was monitored by thin-layer chromatography (TLC) using precoated TLC plates. Iodine chamber and UV lamp were used for visualization of TLC spots.

\section{Experimental}

\section{Synthesis of 3-(4-aminophenylimino)}

\section{indolin-2-one (3)}

Equimolar quantities of isatin (1) and pphenylenediamine (2) were dissolved in ethanol in a $250 \mathrm{ml}$ round bottom flask. The glacial acetic acid (2-3 drops) was added and reaction solution was refluxed for $4 \mathrm{hrs}$. After standing for approximately $24 \mathrm{hr}$ at room temperature, the product was obtained by filtration, washed and recrystallized with ethanol. Yield: $75.59 \%$, m.p. $215-220^{\circ} \mathrm{C}$. 
N-(4-(2-oxoindolin-3-ylideneamino)

phenyl)-2-chloroacetamide (4)

Equimolar quantities of 3-(4aminophenylimino) indolin-2-one (3) and chloroacetylchloride were dissolved in acetonitrile in a $250 \mathrm{ml}$ round bottom flask. The addition of chloroacetylchloride was done in dropwise manner. The anhydrous potassium carbonate was added and reaction mixture was refluxed for 4-5 hrs.

The reaction mixture was filtered and filtrate was vacuum dried. Obtained product was recrystallized with ethanol. Yield: $59.61 \%$, m.p: $235-240^{\circ} \mathrm{C}, \mathrm{R}_{\mathrm{f}}: 0.54$ (n-Hexane: Ethyl acetate; 1:1).

\section{General procedure for synthesis of isatin derivatives (PD1-15)}

Equimolar quantities of N-(4-(2oxoindolin-3-ylideneamino) phenyl)-2chloroacetamide (4) and phenols or primary amines were dissolved in acetonitrile in a $250 \mathrm{ml}$ round bottom flask. The anhydrous potassium carbonate ( 2 mole) was added and reaction solution was refluxed for 12 hrs. The reaction solution was filtered and solvent was removed under reduced pressure. The obtained product was washed with excess of water and purified by recrystallization with ethanol.
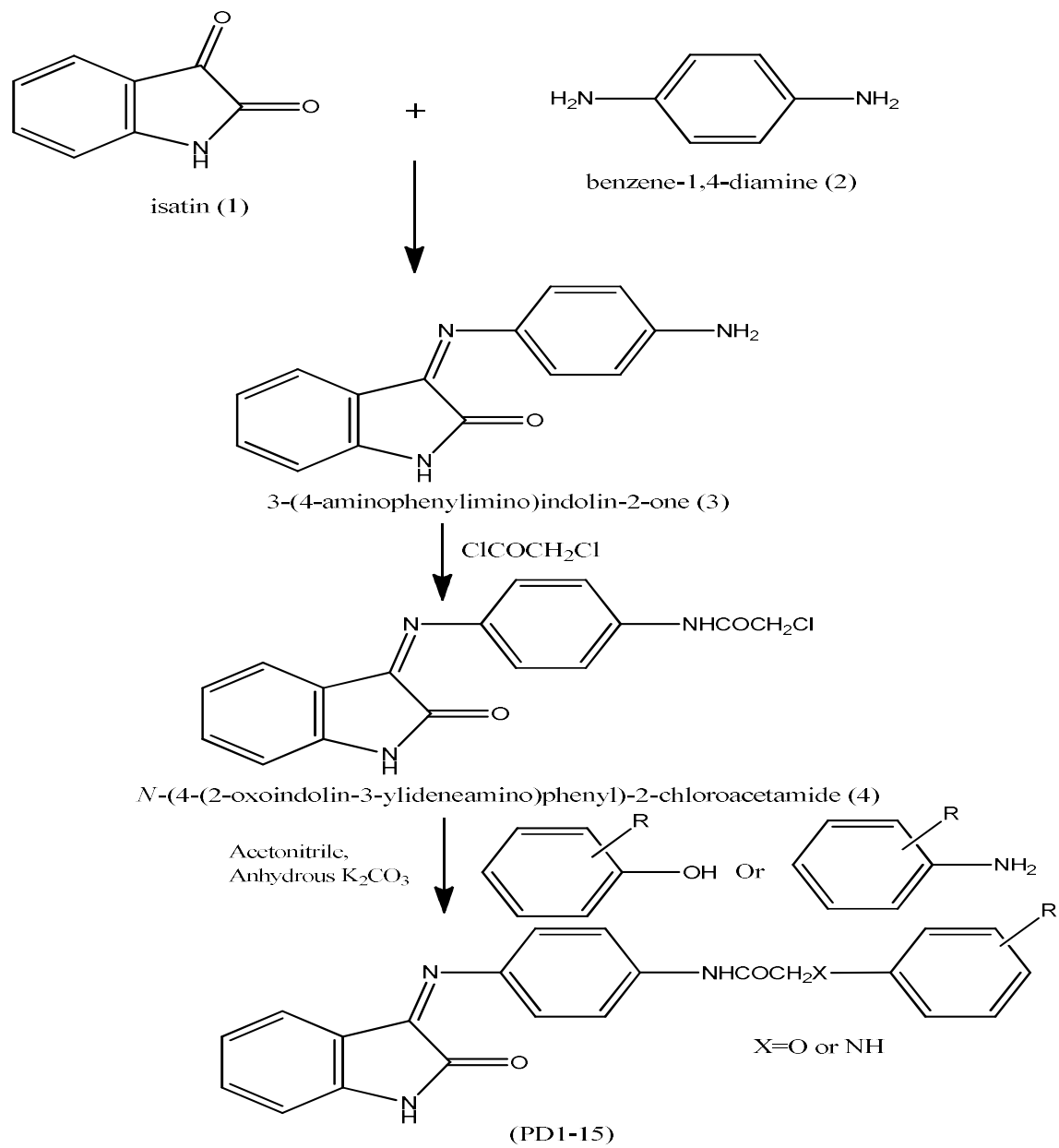

Scheme-1: Synthesis of Isatin derivatives(PD1-15) 
2-(3-bromophenoxy)-N-(4-((2-oxoindolin-

3-ylidene) amino) phenyl) acetamide (PD1)

IR (KBr)cm ${ }^{-1}: 3189$ (N-H Str), 2919 (C-H

Str Ar), 2850 (C-H Str Ali), 1641(C=O

Str), 1367 (C=N Str), 1305 (C-N Str), ${ }^{1} \mathrm{H}-$

NMR (512MHz, $\left.\mathrm{CDCl}_{3}\right)$ (PPM): 10.63 (s, 1H, NH), 7.94 (s, 1H, NH), 7.35-6.56 (m, 12H, Ar-H), 3.84 (s, 2H, $\mathrm{CH}_{2}$ ), Yield: 60.39 \%, m.p.: $255-260{ }^{\circ} \mathrm{C}, \mathrm{R}_{\mathrm{f}}: 0.45$ (n-Hexane:

Ethyl acetate; 1:1).

2-(2, 6-dichlorophenoxy)-N-(4-((2-oxoindolin3-ylidene) amino) phenyl) acetamide (PD2)

IR (KBr)cm ${ }^{-1}$ : 3075 (N-H Str), 2815 (C-H Str Ar), 2750 (C-H Str Ali), 1597(C=O Str), 1475 (C=N Str), 1365(C-N Str), ${ }^{1} \mathrm{H}-$ NMR (500MHz, $\left.\mathrm{CDCl}_{3}\right)$ (PPM): 11.03 (s, 1H, NH), 7.92 (s, 1H, NH), 7.67-6.59 (m, $11 \mathrm{H}, \mathrm{Ar}-\mathrm{H}), 4.12\left(\mathrm{~s}, 2 \mathrm{H}, \mathrm{CH}_{2}\right)$, Yield: $58.98 \%$, m.p: $235-240{ }^{\circ} \mathrm{C}, \mathrm{R}_{\mathrm{f}}: 0.33$ (nHexane: Ethyl acetate; 1:1).

4-((2-oxo-2-((4-((2-oxoindolin-3-ylidene) amino) phenyl) amino) ethyl) amino) benzoic acid (PD3)

IR (KBr)cm ${ }^{-1}: 3270$ (N-H Str), 2890 (C-H Str Ar), 2720 (C-H Str Ali), 1720 (C=O Str), 1410 (C=N Str), 1297 (C-N Str), ${ }^{1} \mathrm{H}-$ NMR (512MHz, $\left.\mathrm{CDCl}_{3}\right)$ (PPM): 11.03 (s, 1H, NH), 09.87 (s, 1H, COOH), 7.92 (s, 1H, NH), 7.91 (s, 1H, NH), 7.34-6.56 (m, 12H, Ar-H), 4.23 (s, 2H, $\mathrm{CH}_{2}$ ), Yield: $55.95 \%$, m.p.: $215-220{ }^{\circ} \mathrm{C}, \mathrm{R}_{\mathrm{f}}: 0.71$ (nHexane: Ethyl acetate; 1:1).
2-(( 3- chlorophenyl) amino)-N- (4- ((2oxoindolin- 3- ylidene) amino) phenyl) acetamide (PD4)

IR (KBr)cm ${ }^{-1}: 3150$ (N-H Str), 2910 (C-H Str Ar), 2720 (C-H Str Ali), 1610 (C=O Str), 1520 (C=N Str), 1430 (C-N Str), ${ }^{1} \mathrm{H}-$ NMR (500 MHz, $\mathrm{CDCl}_{3}$ ) (PPM): 10.70 (s, 1H, NH), $7.92(\mathrm{~s}, 1 \mathrm{H}, \mathrm{NH}), 7.91(\mathrm{~s}, 1 \mathrm{H}$, $\mathrm{NH})$, 7.33-6.56 (m, 12H, Ar-H), 4.23 (s, 2H, $\mathrm{CH}_{2}$ ), Yield: $55.95 \%$, m.p: 210-215 ${ }^{\circ} \mathrm{C}, \mathrm{R}_{\mathrm{f}}: 0.55$ (n-Hexane: Ethyl acetate; 1:1). N-(4-((2-oxoindolin-3-ylidene) amino) phenyl)2-(o-tolylamino) acetamide (PD5)

IR (KBr)cm ${ }^{-1}$ : 3180 (N-H Str), 2850 (C-H Str Ar), 2775 (C-H Str Ali), 1550 (C=O Str), 1375 (C=N Str), 1275(C-N Str), ${ }^{1} \mathrm{H}-$ NMR (500MHz, $\left.\mathrm{CDCl}_{3}\right)$ (PPM): 10.03 (s, 1H, NH), 7.92 (s, 1H, NH), $7.91(\mathrm{~s}, 1 \mathrm{H}$, $\mathrm{NH}), 7.35-6.56(\mathrm{~m}, 12 \mathrm{H}, \mathrm{Ar}-\mathrm{H}), 4.23$ (s, 2H, $\left.\mathrm{CH}_{2}\right), 3.84\left(\mathrm{~s}, 2 \mathrm{H}, \mathrm{CH}_{3}\right)$, Yield: 63.15 \%, m.p: $245-250{ }^{\circ} \mathrm{C}, \mathrm{R}_{\mathrm{f}}: 0.65$ (n-Hexane: Ethyl acetate; $1: 1)$.

2-((2-chlorophenyl) amino)-N-(4-((2-oxoindolin-3ylidene) amino) phenyl) acetamide (PD6)

IR (KBr)cm ${ }^{-1}: 3080$ (N-H Str), 2850 (C-H Str Ar), 2775 (C-H Str Ali), $1710(\mathrm{C}=\mathrm{O}$ Str), 1420 (C=N Str), 1290 (C-N Str), ${ }^{1} \mathrm{H}-$ NMR (500MHz, $\left.\mathrm{CDCl}_{3}\right)$ (PPM): 11.05 (s, 1H, NH), $7.92(\mathrm{~s}, 1 \mathrm{H}, \mathrm{NH}), 7.91(\mathrm{~s}, 1 \mathrm{H}$, $\mathrm{NH}), 7.35-6.56(\mathrm{~m}, 12 \mathrm{H}, \mathrm{Ar}-\mathrm{H}), 4.23$ (s, $2 \mathrm{H}, \mathrm{CH}_{2}$ ), Yield: $67.51 \%$, m.p: $210-215{ }^{\circ} \mathrm{C}$, $\mathrm{R}_{\mathrm{f}}: 0.39$ (n-Hexane: Ethyl acetate; $1: 1$ ). 
2-((3-chloro-4-fluorophenyl) amino)-N-(4((2-oxoindolin-3-ylidene) amino) phenyl) acetamide (PD7)

IR (KBr)cm ${ }^{-1}: 3090$ (N-H Str), 2850 (C-H Str Ar), 2790 (C-H Str Ali), 1750 (C=O Str), 1490 (C=N Str), 1370 (C-N Str), ${ }^{1} \mathrm{H}_{-}$ NMR (500MHz, $\left.\mathrm{CDCl}_{3}\right)$ (PPM): 11.07 (s, 1H, NH), $7.92(\mathrm{~s}, 1 \mathrm{H}, \mathrm{NH}), 7.91(\mathrm{~s}, 1 \mathrm{H}$, NH), 7.28-6.59 (m, 11H, Ar-H), 4.06 (s, $2 \mathrm{H}, \mathrm{CH}_{2}$ ), Yield: $63.82 \%$, m.p: $225-230$ ${ }^{\circ} \mathrm{C}, \mathrm{R}_{\mathrm{f}}: 0.70$ (n-Hexane: Ethyl acetate; 1:1). 2 - ( (2- bromophenyl) amino) - N - (4 - ((2 oxoindolin - 3 - ylidene) amino) phenyl) acetamide (PD8)

IR (KBr) cm ${ }^{-1}: 3280$ (N-H Str), 2875 (C-H Ar), 2790 (C-H Ali), 1650 (C=O Str), 1425 $(\mathrm{C}=\mathrm{N} \quad$ Str $), 1290 \quad$ (C-N $\quad$ Str), ${ }^{1} \mathrm{H}-\mathrm{NMR}$ $\left(500 \mathrm{MHz}, \mathrm{CDCl}_{3}\right)$ (PPM): $11.03(\mathrm{~s}, 1 \mathrm{H}$, $\mathrm{NH}), 8.81(\mathrm{~s}, 1 \mathrm{H}, \mathrm{NH}), 8.14(\mathrm{~s}, 1 \mathrm{H}, \mathrm{NH})$, 7.49-6.71 (m, 12H, Ar-H), $4.12(\mathrm{~s}, 2 \mathrm{H}$, $\mathrm{CH}_{2}$ ), Yield: $59.52 \%$, m.p: $255-260{ }^{\circ} \mathrm{C}, \mathrm{R}_{\mathrm{f}}$ : 0.80 (n-Hexane: Ethyl acetate; 1:1).

2-((2, 3-dimethylphenyl) amino)-N-(4-((2oxoindolin-3-ylidene) amino) phenyl) acetamide (PD9)

IR (KBr)cm ${ }^{-1}$ : 3210 (N-H Str), 2890 (C-H Str Ar), 2690 (C-H Str Ali), 1710 (C=O Str), 1410 (C=N Str), 1290 (C-N Str), ${ }^{1} \mathrm{H}-$ NMR (512MHz, $\left.\mathrm{CDCl}_{3}\right)$ (PPM): 11.07 (s, $1 \mathrm{H}, \mathrm{NH}), 8.81(\mathrm{~s}, 1 \mathrm{H}, \mathrm{NH}), 8.14(\mathrm{~s}, 1 \mathrm{H}$, $\mathrm{NH}), \quad 7.34-6.59(\mathrm{~m}, 11 \mathrm{H}, \operatorname{Ar}-\mathrm{H}), 4.23(\mathrm{~s}$, $\left.2 \mathrm{H}, \mathrm{CH}_{2}\right), 3.96\left(\mathrm{~s}, 3 \mathrm{H}, \mathrm{CH}_{3}\right), 3.62(\mathrm{~s}, 3 \mathrm{H}$, $\mathrm{CH}_{3}$ ), Yield: $60.56 \%$, m.p.: $260-265^{\circ} \mathrm{C}, \mathrm{R}_{\mathrm{f}}$ : 0.25 (n-Hexane: Ethyl acetate; 1:1).
N- (4- ((2 - oxoindolin- 3 - ylidene) amino) phenyl)-2-(p-tolylamino) acetamide (PD10) IR (KBr)cm ${ }^{-1}: 3120$ (N-H Str), 2890 (C-H Str Ar), 2650 (C-H Str Ali), $1710(\mathrm{C}=\mathrm{O}$ Str), 1420 (C=N Str), 1290 (C-N Str), ${ }^{1} \mathrm{H}-$ NMR (512MHz, $\mathrm{CDCl}_{3}$ ) (PPM): 09.93 (s, $1 \mathrm{H}, \mathrm{NH}), 8.81(\mathrm{~s}, 1 \mathrm{H}, \mathrm{NH}), 8.10(\mathrm{~s}, 1 \mathrm{H}$, NH), 7.24-6.59 (m, 12H, Ar-H), 4.06 (s, $\left.2 \mathrm{H}, \mathrm{CH}_{2}\right), 3.59\left(\mathrm{~s}, 3 \mathrm{H}, \mathrm{CH}_{3}\right)$, Yield: 51.53 \%, m.p.: 250-255 ${ }^{\circ} \mathrm{C}, \mathrm{R}_{\mathrm{f}}: 0.60$ (n-Hexane: Ethyl acetate; 1:1).

2-(4-ethylphenoxy)-N-(4-((2-oxoindolin-3ylidene) amino) phenyl) acetamide (PD11) IR (KBr)cm ${ }^{-1}: 3260$ (N-H Str), 2990 (C-H Str Ar), 2890 (C-H Str Ali), 1310 (C-N Str), 1750 (C=O Str), 1390 (C=N Str), ${ }^{1} \mathrm{H}-$ NMR (512MHz, $\mathrm{CDCl}_{3}$ ) (PPM): 10.87 (s, 1H, NH), 7.48 (s, 1H, NH), 7.47-6.58 (m, 12H, Ar-H), 4.22 (s, 2H, $\mathrm{CH}_{2}$ ), 2.61- 2.20 (m, $\mathrm{CH}_{2} \mathrm{CH}_{3}$ ), Yield: $69.05 \%$, m.p.: 230$235{ }^{\circ} \mathrm{C}, \mathrm{R}_{\mathrm{f}}: 0.81$ (n-Hexane: Ethyl acetate; $1: 1)$.

2-(2, 6-dimethylphenoxy)-N-(4-((2-oxoindolin3-ylidene) amino) phenyl) acetamide (PD12) IR (KBr) $\mathrm{cm}^{-1}$ : 3270 (N-H Str), 2960 (C-H Str Ar), 2810 (C-H Str Ali), 1590 (C=O Str), 1480 (C=N Str), 1360 (C-N Str), ${ }^{1} \mathrm{H}-$ NMR (500MHz, $\mathrm{CDCl}_{3}$ ) (PPM): 11.07 (s, $1 \mathrm{H}, \mathrm{NH}), 8.00(\mathrm{~s}, 1 \mathrm{H}, \mathrm{NH}), 7.94-6.51$ (m, $11 \mathrm{H}, \mathrm{Ar}-\mathrm{H}), 4.23$ (s, 2H, $\left.\mathrm{CH}_{2}\right), 3.81(\mathrm{~s}, 3 \mathrm{H}$, $\mathrm{CH}_{3}$ ), 3.58 (s, 3H, $\mathrm{CH}_{3}$ ), Yield: $52.95 \%$, m.p: $240-245{ }^{\circ} \mathrm{C}, \mathrm{R}_{\mathrm{f}}: 0.75$ (n-Hexane: Ethyl acetate; 1:1). 
2- (4- methoxyphenoxy) - N - (4- ((2oxoindolin-3-ylidene) amino) phenyl) acetamide (PD13)

IR (KBr)cm ${ }^{-1}: 3280$ (N-H Str), 2890 (C-H Str Ar), 2810 (C-H Str Ali), 1425 (C=N Str), 1235 (C-N Str), 1450 (C=O Str), ${ }^{1} \mathrm{H}$ NMR (500MHz, $\left.\mathrm{CDCl}_{3}\right)$ (PPM): 10.63 (s, $1 \mathrm{H}, \mathrm{NH}), 8.14$ (s, 1H, NH), 7.77-6.58 (m, 12H, Ar-H), 3.79 (s, 2H, $\left.\mathrm{CH}_{2}\right), 4.80(\mathrm{~s}, 3 \mathrm{H}$, $\mathrm{OCH}_{3}$ ),Yield: $60.43 \%$, m.p: $220-225{ }^{\circ} \mathrm{C}$, $\mathrm{R}_{\mathrm{f}}: 0.61$ (n-Hexane: Ethyl acetate; 1:1).

2-(2-nitrophenoxy)-N-(4-((2-oxoindolin-3ylidene) amino) phenyl) acetamide (PD14) IR (KBr)cm ${ }^{-1}: 3210$ (N-H Str), 2850 (C-H Str Ali), 2810 (C-H Str Ar), 1690 (C=O Str), 1370 (C=N Str), 1190 (C-N Str), ${ }^{1} \mathrm{H}-$ NMR (500MHz, $\left.\mathrm{CDCl}_{3}\right)$ (PPM): 10.63 (s, $1 \mathrm{H}, \mathrm{NH}), 8.14$ (s, 1H, NH), 7.10-6.59 (m, 12H, Ar-H), 4.23 (s, 2H, $\mathrm{CH}_{2}$ ), Yield: 58.95 \%, m.p: $215-220{ }^{\circ} \mathrm{C}, \mathrm{R}_{\mathrm{f}}: 0.83$ (n-Hexane: Ethyl acetate; 1:1).

2-(2, 5 - dimethylphenoxy $)-\mathrm{N}-(4-((2-$ oxoindolin-3-ylidene) amino) phenyl) acetamide (PD15)

IR (KBr)cm ${ }^{-1}: 3290$ (N-H Str), 2950 (C-H Str Ar), 2870 (C-H Str Ali),1710 (C=O Str), 1430 (C=N Str), 1350 (C-N Str), ${ }^{1} \mathrm{H}$ NMR (500MHz, $\left.\mathrm{CDCl}_{3}\right)$ (PPM): 10.07 (s, $1 \mathrm{H}, \mathrm{NH}), 8.14$ (s, 1H, NH), 7.57-6.59 (m, $11 \mathrm{H}, \mathrm{Ar}-\mathrm{H}), 4.23$ (s, 2H, $\left.\mathrm{CH}_{2}\right), 3.81(\mathrm{~s}, 3 \mathrm{H}$, $\left.\mathrm{CH}_{3}\right), 3.78\left(\mathrm{~s}, 3 \mathrm{H}, \mathrm{CH}_{3}\right)$, Yield: $55.51 \%$, m.p: $255-260{ }^{\circ} \mathrm{C}, \mathrm{R}_{\mathrm{f}}: 0.77$ (n-Hexane: Ethyl acetate; 1:1).
Molecular properties prediction for test compounds (PD1-PD15)

A set of molecular properties molar refractivity [14], topological surface area [12] and $\log \mathrm{P}[\mathbf{1 3}]$ were computed for the target compounds as well as two standard drugs Ciprofloxacin and Fluconazole using Chem 3D Ultra version 12.0, software programs. The observations are depicted in Table 1.

\section{In vitro antimicrobial activity}

The antimicrobial assay is based upon a comparison of the inhibition of growth of micro-organism by measured concentrations of the antimicrobial agents to be examined with that procedure by known concentration of standard preparation of the antibiotics having a known activity [11, 15]. Four strains of bacteria and four strains of fungi were taken as the test organism. In order to evaluate the antibacterial spectrum of the test compounds, strains of Bacillus subtillis (MTCC 441), Escherichia coli (MTCC 1573), Staphylococcus aureus (MTCC1430), and Klebsiella pneumoniae (MTCC 618), were procured. For antifungal spectrum strains of Aspergillus niger (MTCC 2546), Rhizopus oryzae (MTCC 2775), Candida albicans (MTCC 183) and Penicillium chrysogenum (MTCC161), were procured as pure culture from Institute of Microbial Technology, Chandigarh. Growth media were prepared 
in accordance with the direction laid down in the package inserted. The test organisms were inoculated in nutrient broth. A definite volume of this suspension was mixed with nutrient agar (cooled to $40^{\circ} \mathrm{C}$ ). Nutrient agar was poured into petridishes to obtain a uniform thickness. The surface of agar plates was pierced using a sterile cork borer. The prepared wells were filled with equal volumes of a solution of antimicrobial agents PD1-PD15. After a period of pre incubation diffusion, the plates were incubated face up for a definite time and at the specific temperature for that strain. The diameters of zones of inhibition were measured (Table 2 and Table 3).

Table 1: Molecular properties of the test compounds (PD1-PD15)

\begin{tabular}{|c|c|c|c|c|c|}
\hline Compound Code & Molecular Formula & $\mathbf{M W}^{\mathbf{x}}$ & $\mathbf{M R}^{\mathbf{y}}$ & tPSA $^{\mathrm{z}}$ & $\log P$ \\
\hline PD1 & $\mathrm{C}_{22} \mathrm{H}_{16} \mathrm{BrN}_{3} \mathrm{O}_{3}$ & 450.28 & 112.99 & 79.79 & 3.68 \\
\hline PD2 & $\mathrm{C}_{22} \mathrm{H}_{15} \mathrm{Cl}_{2} \mathrm{~N}_{3} \mathrm{O}_{3}$ & 440.28 & 114.51 & 79.79 & 3.97 \\
\hline PD3 & $\mathrm{C}_{23} \mathrm{H}_{18} \mathrm{~N}_{4} \mathrm{O}_{4}$ & 414.41 & 114.05 & 119.89 & 2.03 \\
\hline PD4 & $\mathrm{C}_{22} \mathrm{H}_{17} \mathrm{CIN}_{4} \mathrm{O}_{2}$ & 404.85 & 111.84 & 82.59 & 3.03 \\
\hline PD5 & $\mathrm{C}_{23} \mathrm{H}_{20} \mathrm{~N}_{4} \mathrm{O}_{2}$ & 384.43 & 113.14 & 82.59 & 2.96 \\
\hline PD6 & $\mathrm{C}_{22} \mathrm{H}_{17} \mathrm{CIN}_{4} \mathrm{O}_{2}$ & 404.10 & 111.84 & 82.59 & 3.03 \\
\hline PD7 & $\mathrm{C}_{22} \mathrm{H}_{16} \mathrm{ClFN}_{4} \mathrm{O}_{2}$ & 422.84 & 112.25 & 82.59 & 3.19 \\
\hline PD8 & $\mathrm{C}_{22} \mathrm{H}_{17} \mathrm{BrN}_{4} \mathrm{O}_{2}$ & 449.30 & 114.93 & 82.59 & 3.30 \\
\hline PD9 & $\mathrm{C}_{24} \mathrm{H}_{22} \mathrm{~N}_{4} \mathrm{O}_{2}$ & 398.46 & 119.03 & 82.59 & 3.45 \\
\hline PD10 & $\mathrm{C}_{23} \mathrm{H}_{20} \mathrm{~N}_{4} \mathrm{O}_{2}$ & 384.43 & 113.14 & 82.59 & 2.96 \\
\hline PD11 & $\mathrm{C}_{24} \mathrm{H}_{21} \mathrm{~N}_{3} \mathrm{O}_{3}$ & 399.44 & 115.79 & 79.79 & 3.75 \\
\hline PD12 & $\mathrm{C}_{24} \mathrm{H}_{21} \mathrm{~N}_{3} \mathrm{O}_{3}$ & 399.44 & 117.09 & 79.79 & 3.82 \\
\hline PD13 & $\mathrm{C}_{23} \mathrm{H}_{19} \mathrm{~N}_{3} \mathrm{O}_{4}$ & 401.41 & 112.55 & 89.02 & 2.72 \\
\hline PD14 & $\mathrm{C}_{22} \mathrm{H}_{16} \mathrm{~N}_{4} \mathrm{O}_{5}$ & 416.39 & $\mathrm{NC}$ & 131.60 & 2.45 \\
\hline PD15 & $\mathrm{C}_{24} \mathrm{H}_{21} \mathrm{~N}_{3} \mathrm{O}_{3}$ & 399.44 & 117.09 & 79.79 & 3.82 \\
\hline Ciprofloxacin & $\mathrm{C}_{17} \mathrm{H}_{18} \mathrm{FN}_{3} \mathrm{O}_{3}$ & 331.34 & 89.39 & 72.88 & 1.32 \\
\hline Fluconazole & $\mathrm{C}_{13} \mathrm{H}_{12} \mathrm{~F}_{2} \mathrm{~N}_{6} \mathrm{O}$ & 306.27 & 78.46 & 76.15 & 0.99 \\
\hline
\end{tabular}

Abbreviations: x-Molecular weight, y-Molar refractivity, z-Topological polar surface area, NC-Not calculated

Table 2: Antibacterial Activity of Isatin Derivatives

\begin{tabular}{|c|c|c|c|c|c|}
\hline \multirow{3}{*}{$\begin{array}{c}\text { Compound } \\
\text { Code }\end{array}$} & \multirow{3}{*}{$\begin{array}{c}\text { Conc. } \\
(\mu \mathrm{g} / \mathrm{ml})\end{array}$} & \multicolumn{4}{|c|}{ Zone of inhibition (mm) } \\
\hline & & \multicolumn{2}{|c|}{ Gram +ve } & \multicolumn{2}{|c|}{ Gram -ve } \\
\hline & & B. subtilis & S. aureus & E. coli & K. pneumoniae \\
\hline PD1 & 200 & 16 & 16 & 14 & 15 \\
\hline PD2 & 200 & 17 & 16 & 16 & 16 \\
\hline PD3 & 200 & 13 & 14 & 14 & 12 \\
\hline PD4 & 200 & 16 & 16 & 16 & 17 \\
\hline PD5 & 200 & 15 & 16 & 14 & 15 \\
\hline PD6 & 200 & 24 & 24 & 23 & 22 \\
\hline PD7 & 200 & 17 & 17 & 16 & 16 \\
\hline PD8 & 200 & 23 & 22 & 21 & 21 \\
\hline PD9 & 200 & 16 & 15 & 14 & 15 \\
\hline PD10 & 200 & 15 & 16 & 14 & 14 \\
\hline PD11 & 200 & 12 & 14 & 15 & 13 \\
\hline PD12 & 200 & 14 & 15 & 14 & 15 \\
\hline PD13 & 200 & 17 & 15 & 15 & 16 \\
\hline PD14 & 200 & 22 & 21 & 21 & 20 \\
\hline PD15 & 200 & 16 & 14 & 15 & 14 \\
\hline Ciprofloxacin & 200 & 26 & 27 & 25 & 25 \\
\hline DMF & & - & - & - & - \\
\hline
\end{tabular}

Derivatives found with zone of inhibition above $18 \mathrm{~mm}$ are active. Standard - Ciprofloxacin, Control - DMF 
Table 3: Antifungal Activity of Isatin Derivatives

\begin{tabular}{|c|c|c|c|c|c|}
\hline \multirow{2}{*}{$\begin{array}{l}\text { Compound } \\
\text { code }\end{array}$} & \multirow{2}{*}{$\begin{array}{l}\text { Conc. } \\
(\mu \mathrm{g} / \mathrm{ml})\end{array}$} & \multicolumn{4}{|c|}{ Zone of inhibition (mm) } \\
\hline & & A. niger & R. oryzae & C. albicans & P. chrysogenum \\
\hline PD1 & 200 & 16 & 15 & 15 & 14 \\
\hline PD2 & 200 & 21 & 20 & 20 & 20 \\
\hline PD3 & 200 & 11 & 14 & 14 & 13 \\
\hline PD4 & 200 & 16 & 17 & 16 & 15 \\
\hline PD5 & 200 & 14 & 13 & 14 & 14 \\
\hline PD6 & 200 & 16 & 17 & 16 & 16 \\
\hline PD7 & 200 & 22 & 21 & 21 & 21 \\
\hline PD8 & 200 & 16 & 15 & 14 & 14 \\
\hline PD9 & 200 & 11 & 14 & 13 & 14 \\
\hline PD10 & 200 & 12 & 11 & 15 & 14 \\
\hline PD11 & 200 & 10 & 11 & 13 & 12 \\
\hline PD12 & 200 & 12 & 13 & 13 & 14 \\
\hline PD13 & 200 & 16 & 15 & 14 & 14 \\
\hline PD14 & 200 & 20 & 19 & 19 & 19 \\
\hline PD15 & 200 & 13 & 14 & 13 & 15 \\
\hline Fluconazole & 200 & 23 & 22 & 24 & 22 \\
\hline DMF & & - & - & - & - \\
\hline
\end{tabular}

Derivatives found with zone of inhibition above $18 \mathrm{~mm}$ are active. Standard - Fluconazole, Control - DMF

\section{RESULTS AND DISCUSSION}

Synthesis of isatin derivatives (PD1-15) has shown in Scheme 1. All the derivatives were obtained in good yield. The structures of test compounds were confirmed on the basis of IR and ${ }^{1} \mathrm{H}$ NMR method. The molecular properties of test compounds were calculated. Most of the test compounds have molar refractivity between 119.03-111.84. Topological polar surface area (tPSA) values for the test compounds were found within 79.79-131.60, which is a surface sum over all polar atoms, primarily oxygen and nitrogen, also their attachment to the hydrogen atoms that helps the compound permeation through the cell. The Log P values of test compound were 2.03-3.97. The test compounds were tested for their antimicrobial activity against different bacteria and fungi. The test compounds containing halogen atoms like chloro, bromo, fluoro and nitro group (electron withdrawing groups) were showing significant antibacterial and antifungal activity, which depicts that halogen atoms (electron withdrawing group) have an important role in antimicrobial activity. Among all, test compound PD6, PD8, and PD14 possess potent antibacterial activity, whereas PD2, PD7 and PD14 possess potent antifungal activity.

\section{CONCLUSION}

In present study, Isatin derivatives were synthesized and evaluated for antimicrobial activities. It has been found that isatin derivatives can be used as a lead in development of novel antimicrobial agents.

\section{ACKNOWLEDGEMENT}

The authors are thankful to Prof. M.P. Pandey, Vice Chancellor, IFTM University, Moradabad (U.P) India and Prof. Ranjit 
Singh, Pro Vice Chancellor, Shobhit University, Gangoh, Saharanpur (U.P) India for their unbound support and valuable help during the research work. I also thanks to Microbial Type Culture Collection and Gene Bank (MTCC), Institute of Microbial Technology, Chandigarh for providing microbial strains.

\section{REFERENCES}

[1] Mogilaiah, K.; Ramesh Babu, H.; BabuRao, R: Synthesis and antibacterial activity of some new $[1,3,4]$ oxadiazino $[6,5-b]$ indole and acetophenonehydrazones containing 1 , 8 - naphthyridine moiety, Indian Journal of Chemistry 2001; 40B: 127074.

[2] Chavan, P.; Mane, A. S.; Shingare, M. S: Synthesis of new O, Odialkyl-O-coumarino-phosphorothioates and their pesticidal bioassay against Helicoverpa armigera, Indian Journal of Chemistry 2001; 40B: 339-41.

[3] Da Silva, J.F.M.; Garden, S.J.; Pinto, A.C.: The chemistry of isatins: a review from 1975 to 1999 , Journal of the Brazilian Chemical Society $2001 ; 12:$ 273-324.

[4] Mazhilis, L.I.; Terent, P.B.; Boltin, V.A.: Synthesis of indole and oxindole derivatives incorporating pyrrolidino, pyrrolo or imidazolo moieties, Chemistry of Heterocyclic Compounds, 1989; 25: 50-54.

[5] Pandeya, S.N., Smitha, S., Jyoti, M., Sridhar, S.K.: Biological activities of isatin and its derivatives. Acta Pharm. 2005; 55: 27-46.

[6] Lian-Shun, F., Ming-Liang, L., Shu, Z., Yun, C., Wang, B., Yi-Bin, Z., Kai, L., Yan, G., Hui-Yuan, G., Chun-Ling, X.: Synthesis and in vitro antimycobacterial activity of 8-OCH3ciprofloxacin methylene and ethylene isatin derivatives. Eur. J. Med. Chem. 2011: 46: 341-348.

[7] Praveen, C., Ayyanar, A., Perumal, P.T. Practical synthesis, anticonvulsant, and antimicrobial activity of $\mathrm{N}$ - allyl and N-propargyl di (indolyl) indolin-2-ones. Bioorg. Med. Chem. Lett. 2011; 21: 4072-4077.

[8] Amalraj, A., Raghunathan, R., SrideviKumari, M.R., Raman, N.: Synthesis, antimicrobial and antifungal activity of a new class of spiro pyrrolidines. Bioorg. Med. Chem. 2003; 11: 407-419.

[9] Sanjana C., Meenakumari S., Munusamy T., Jagadeshwar R. T., Vairamani $\mathrm{M}$ and Pachaiappan R.: A facile approach to the isolation of proteins in Ferula asafoetida and their enzyme stabilizing, antimicrobial and anti-oxidant activity, 
Int. J. Biol. Macromol., 2017; 102: 1211-1219.

[10] Naif O Al-Harbi., Saleh A. B., Ahmed A F, Moutasem S A and Abd El-Galil E. A, Antiparkinsonism, hypoglycemic and anti-microbial activities of new poly fused ring heterocyclic candidates, Int. J. Biol. Macromol., 2013; 57: 165-173.

[11] Indian Pharmacopoeia 2007, Vol I, $35-48$.

[12] Pajouhesh H, Lenz GR.: Medicinal chemical properties of successful central nervous system drugs NeuroRx., 2005; 2(4): 541-553.

[13] Kumar, S., Wahi, A. K., \& Singh, R.: Synthesis, computational studies and preliminary pharmacological evaluation of 2 [4-(aryl substituted) piperazin-1yl] N, N-diphenylacetamides as potential antipsychotics. European Journal of Medicinal Chemistry, 2011; 46(9): 4753-4759.

[14] Kumar, S., Wahi, A. K., \& Singh, R.: Synthesis, computational studies and preliminary pharmacological evaluation of 2-[4-(aryl substituted) piperazin-1-yl]-N-benzylacetamides as potential antipsychotics. Arabian Journal of Chemistry, 2016; 9: S1450-S1456.

[15] Mohit, Kuma,r S., Kumar, S., \& Hashim, S. R.: New Phenoxy Benzoyl Methane Derivatives: Synthesis and Evaluation of the Antimicrobial Potential, Journal of Drug Delivery \& Therapeutics, 2019; 9(2-s): 402-405. 